\title{
Robotics on a mission
}

\author{
As nations come together in Tokyo next summer to celebrate the spirit of human potential in the 2020 Olympic \\ Games, they will have a chance to take part in another international competition hosted by Japan soon after, this \\ time with challenges designed for robot contenders.
}

\begin{abstract}
he World Robot Summit, which will take place during two main events in August and October next year, will test the potential of robots in a range of challenging competitions. We spoke with Satoshi Tadokoro from Tohoku University, who is leading the disaster robotics competition. A preliminary version leading up to this competition was held in 2018 (pictured) and findings were recently reported ${ }^{1}$.
\end{abstract}

Previous large-scale robot competitions such as the DARPA Grand Challenge ${ }^{2}$ have been important for driving innovation in robotics and also in public perception of robotics. A large-scale challenge for disaster robotics could play a similarly stimulating role but can only be carried out as a government initiative or with substantial government support, you recently wrote ${ }^{1}$. Can you say more about this? It is difficult for private companies to sustainably support projects in preventing and responding to natural or man-made disasters. Tax payers should expect governments to take responsibility in this area. History shows that major disasters increased citizens' anxiety, and have driven to the collapse of governments. I believe it is the duty of governments and inter-government organizations like the United Nations to encourage development of technologies to prevent and mitigate disasters.

Was the vision for the competition influenced by the Fukushima Daiichi nuclear power plant accident in 2011?

Disasters occur frequently, everywhere in the world. Natural and man-made disasters like the Fukushima Daiichi accident as well as huge earthquakes, floods, storms, landslides and so on have had a major impact on countries. The importance of organizing competitions to promote research and development was pointed out as an action to be taken after Fukushima. This was a starting point for organizing the World Robot Summit Disaster Robotics Category, which will take place in a test field located in the Fukushima prefecture.

Are you encouraged by the results of the preliminary robotics competition? There were in total 37 teams from many different countries participating in the

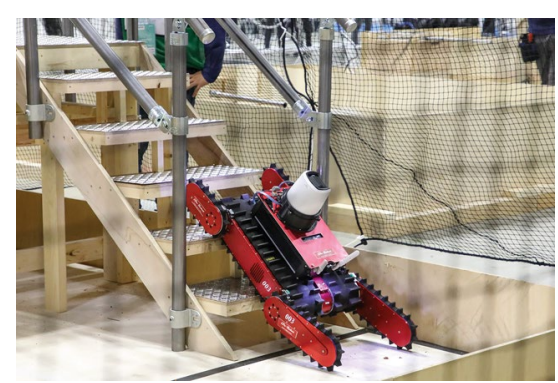

A mobility task in the 2018 preliminary Disaster Robotics Challenge. Credit: Hiroko Tadokoro

preliminary competition and they showed potential capabilities in realistic situations such as routine inspection and equipment regulation. I expect that the teams can develop their robot approaches further and demonstrate more practical performance in 2020 .

The results indicate that robots were generally good at visual reports, but manipulation tasks such as opening and closing valves and operating a lever handle were harder, making teams strategically avoid such tasks. What can be done to encourage better performance at manipulation tasks? Previous robotics competitions typically used very loose valves that could be opened by small torque, but such a largely scientific challenge is completely different from the real problems that need to be solved for future innovation. Therefore, the Disaster Robotics Category adopts tasks that are as realistic as possible, with 'real', tight valves. Some teams had to give up this difficult challenge in the preliminary rounds of 2018 , but now that they understand the level of difficulty I believe they can solve these problems in 2020 . In order to promote the teams' efforts in this direction, the organizers will adjust the score system so that participating teams must try to solve difficult tasks such as manipulation to achieve a high score.

What level of autonomous capabilities versus remote control do you think is needed?

From the viewpoint of users, it does not matter whether the robots are autonomous or tele-operated. The important points are that (1) robots can replace humans in certain tasks to lower the risks and reduce difficulties in extreme conditions of disaster, (2) robots have better or equivalent performance to humans in the case of an unexpected emergency, and (3) robots can reduce the cost to society. Teleoperation cannot be avoided in unexpected situations. Therefore, seamless transitions to and from autonomous work and tele-operated work by sharing roles of humans and robots are essential. At the same time, cooperation of remote and on-site control, and processing in the cloud and at the edge, by ad hoc wireless communication is important.

Submissions are now open until 31 August this year. Teams can enter the competition with one unmanned ground vehicle (UGV) and one small unmanned aerial vehicle (UAV). What are the advantages of such a combination?

UAVs have quick accessibility to high places but cannot have many sensors or manipulation capabilities. Contact with structures results in UAV crashes. UGVs can carry various sensors for non-destructive inspection and manipulators can open and close valves. But accessibility of UGVs is limited because their mobility is completely inferior to human workers. Therefore, role sharing of various robot systems (not only conventional UAVs and UGVs) is needed.

The aim is to organize the World Robot Summit every four years, hosted by different countries, following the schedule of the Olympic Games. Is there already interest from other countries to hold the next robot 'Olympics' in 2024, and how will other governments be encouraged?

I heard that some countries are interested in continuing this 'Olympics' as the second and third host countries. It is a challenge for all of humanity to ensure innovation in response to and prevention of disaster. This is one of the most significant social issues that governments can be expected to tackle on an international level.

\section{Interview by Liesbeth Venema}

Published online: 9 August 2019

https://doi.org/10.1038/s42256-019-0081-9

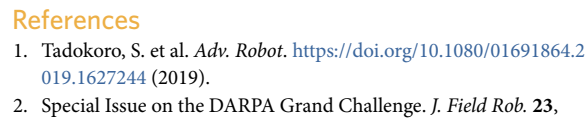

